\title{
Tobramycin exposure from active calcium sulfate bone graft substitute
}

Françoise Livio ${ }^{1 *}$, Peter Wahl ${ }^{2}$, Chantal Csajka ${ }^{1,3}$, Emanuel Gautier ${ }^{2}$ and Thierry Buclin ${ }^{1}$

\begin{abstract}
Background: Bone graft substitute such as calcium sulfate are frequently used as carrier material for local antimicrobial therapy in orthopedic surgery. This study aimed to assess the systemic absorption and disposition of tobramycin in patients treated with a tobramycin-laden bone graft substitute (Osteoset $\left.{ }^{\oplus} \mathrm{T}\right)$.

Methods: Nine blood samples were taken from 12 patients over 10 days after Osteoset ${ }^{\oplus}$ T surgical implantation. Tobramycin concentration was measured by fluorescence polarization. Population pharmacokinetic analysis was performed using NONMEM to assess the average value and variability (CV) of pharmacokinetic parameters. Bioavailability (F) was assessed by equating clearance (CL) with creatinine clearance (Cockcroft $\mathrm{CLCr}$ ). Based on the final model, simulations with various doses and renal function levels were performed. (ClinicalTrials.gov number, NCT01938417).
\end{abstract}

Results: The patients were $52+/-20$ years old, their mean body weight was $73+/-17 \mathrm{~kg}$ and their mean $\mathrm{CLCr}$ was $119+/-55 \mathrm{~mL} / \mathrm{min}$. Either $10 \mathrm{~g}$ or $20 \mathrm{~g}$ Osteoset $^{\oplus} \mathrm{T}$ with $4 \%$ tobramycin sulfate was implanted in various sites. Concentration profiles remained low and consistent with absorption rate-limited first-order release, while showing important variability. With $\mathrm{CL}$ equated to $\mathrm{CLCr}$, mean absorption rate constant (ka) was $0.06 \mathrm{~h}-1, \mathrm{~F}$ was $63 \%$ or $32 \%$ (CV 74\%) for 10 and $20 \mathrm{~g}$ Osteoset $^{\oplus}$ T respectively, and volume of distribution (V) was $16.6 \mathrm{~L}$ (CV 89\%). Simulations predicted sustained high, potentially toxic concentrations with $10 \mathrm{~g}, 30 \mathrm{~g}$ and $50 \mathrm{~g}$ Osteoset ${ }^{\circledR} \mathrm{T}$ for $\mathrm{CLCr}$ values below 10, 20 and $30 \mathrm{~mL} / \mathrm{min}$, respectively.

Conclusions: Osteoset ${ }^{\oplus} T$ does not raise toxicity concerns in subjects without significant renal failure. The risk/benefit ratio might turn unfavorable in case of severe renal failure, even after standard dose implantation.

Keywords: Tobramycin, Bone graft substitute, Pharmacokinetics, Renal failure

\section{Background}

Since the end of the sixties, bone cement such as polymethyl methacrylate (PMMA) and bone graft substitute such as calcium sulfate are frequently used as carrier material for local antimicrobial therapy in orthopedic surgery for osteomyelitis, infected arthroplasty, soft tissue infections or prophylaxis. Unlike PMMA, calcium sulfate is resorbable, thus obviating the need for surgical removal. Osteoset $^{\oplus} \mathrm{T}$ (Wright Medical Technology Inc, Arlington, TN, USA) is one of these products; it contains $4 \%$ tobramycin sulfate in calcium sulfate pellets. Most data on Osteoset $^{\circ} \mathrm{T}$ come from animal studies, where high local and low systemic tobramycin concentrations have been observed [1]. No pharmacokinetic (PK) observations with

\footnotetext{
* Correspondence: francoise.livio@chuv.ch

${ }^{1}$ Division of Clinical Pharmacology, Biomedicine, Department of Laboratories, Centre Hospitalier Universitaire Vaudois, Lausanne 1011, Switzerland Full list of author information is available at the end of the article
}

patients treated with Osteoset ${ }^{\circ} \mathrm{T}$ have been published so far, despite well known concentration-related potential toxicity of aminoglycosides. Only concentrations have been described in patients implanted with a tobramycinladen polymer carrier material (Simplex ${ }^{\mathrm{Tm}} \mathrm{P}$ Bone Cement with Tobramycin) showing high tobramycin levels at the operative site but low systemic absorption [2].

The aim of this study was to develop a population PK model for tobramycin in patients treated with an active calcium sulfate bone substitute and to predict tobramycin systemic exposure under various dose and renal function levels.

\section{Method}

\section{Patients and sampling}

The data were collected prospectively between October 2006 and March 2008 from all adult patients treated with 
Osteoset $^{\oplus} \mathrm{T}$ in our orthopedic surgery department. Osteoset $^{\oplus} \mathrm{T}$ was implanted whenever estimated a useful adjunct to standard therapy for bone, soft tissues and prosthetic joint infections requiring surgical debridement. Either $10 \mathrm{~g}$ or $20 \mathrm{~g}$ of Osteoset ${ }^{\oplus} \mathrm{T}$ with $4 \%$ tobramycin sulfate were implanted, representing 262 or $524 \mathrm{mg}$ tobramycin repectively taking into account the salt factor (tobramycin/tobramycin sulfate $=0.655$ ). Additional intravenous aminoglycosides were not used in these patients. No wound drains were used.

Blood samples were taken at 3 h, 6 h, 12 h, 24 h, 48 h and on days 3, 5, 7 and 10 after implantation. Whenever a tourniquet had been used during the operation, its release-time was taken into account instead of implantation time. Blood was sampled in BD Vacutainer ${ }^{\ominus}$ SST II Advance (Becton Dickinson AG, Allschwil, Switzerland) serum separator tubes.

The study protocol was approved by the Institutional Review Board of Fribourg Cantonal Hospital and all subjects gave their written informed consent. This study was registered in ClinicalTrials.gov under code number NCT01938417.

\section{Drug assay}

Tobramycin concentrations were measured on a COBAS INTEGRA 800 (Roche Diagnostics GmbH, Mannheim, Germany) fluorescence polarization detector, using a standard tobramycin measurement kit (ref. 20737925). The lower limit of quantification (LOQ) of the assay was $0.1 \mathrm{mg} / \mathrm{L}$. Coefficient of variation $(\mathrm{CV})$ within runs was $1.7 \%$ at $2.2 \mathrm{mg} / \mathrm{L}$, while $\mathrm{CV}$ between runs were $6.2 \%, 2.6 \%$ and $2.5 \%$ at $0.9 \mathrm{mg} / \mathrm{L}, 2.8 \mathrm{mg} / \mathrm{L}$ and $4.5 \mathrm{mg} / \mathrm{L}$, respectively. Accuracy was monthly checked by participating to the external quality control scheme provided by LGC Standards for antibiotics (LGC Standards Proficiency Testing, Bury, UK). Assays were processed within 6 hours after sampling.

\section{Population pharmacokinetic analysis}

The pharmacokinetic analysis was performed using nonlinear mixed-effects modeling with the NONMEM computer program (version VI) [3]. Models were fitted to the data with the first-order conditional estimation with interaction (FOCE INTER) method. One and two compartment models with first-order absorption were compared. The first tobramycin concentration below the LOQ was set to half of the LOQ value and subsequent points below the LOQ were dropped out. Average value and variability of clearance (CL), volume of distribution (V) and absorption rate constant $\left(\mathrm{k}_{\mathrm{a}}\right)$ were assessed. As aminoglycosides are known to be completely and exclusively eliminated by glomerular filtration, tobramycin CL was equated to the creatinine clearance $\left(\mathrm{CL}_{\mathrm{cr}}\right)$ value, thus enabling the estimation of absolute bioavailability $(F)$ in the absence of intravenous injection. $\mathrm{CL}_{\mathrm{cr}}$ was estimated with the Cockcroft formula, based on patient's serum creatinine, body weight, and sex [4], with linear interpolation for days without creatinine measurement. The residual variability was adequately modeled using an additive plus proportional error model. The final model was then developed by testing in a stepwise fashion the potential influence of sex, age, body weight, Osteoset $\mathrm{T}$ quantity and implantation site on the pharmacokinetic parameters.

Based on the final average parameters and variability, simulations using various tobramycin doses (262 mg; $786 \mathrm{mg} ; 1310 \mathrm{mg})$ at various $\mathrm{CL}_{\mathrm{cr}}$ levels $(120,90,60,30$, 20 and $10 \mathrm{~mL} / \mathrm{min}$ ) were performed, using 2000 virtual patients each to predict the doses associated with concentrations exceeding the $2 \mathrm{mg} / \mathrm{L}$ threshold over a prolonged period of time ( 5 days or more).

\section{Results}

Twelve patients ( 7 males, 5 females) were included in this observational study. Their mean age was 52 years (range 19-82, SD \pm 20$)$, body weight $73 \mathrm{~kg}(53-116, \pm 17)$, and $\mathrm{CL}_{\mathrm{cr}} 119 \mathrm{~mL} / \mathrm{min}(34-288, \pm 55)$.

Osteoset $^{\oplus} \mathrm{T}$ implantation sites were tibia/fibula (6 patients), hip (2), calcaneum (2), femur (1) and lumbar spine (1), either for established infections (9) or prophylaxis (3). Eight patients were implanted $10 \mathrm{~g}$ Osteoset $^{\oplus} \mathrm{T}$ containing $262 \mathrm{mg}$ tobramycin, while 4 were implanted $20 \mathrm{~g}$ Osteoset $^{\oplus} \mathrm{T}$ containing $524 \mathrm{mg}$ tobramycin.

Osteoset $^{\oplus} \mathrm{T}$ was well tolerated; further clinical details have been presented elsewhere [5]. Systemic tobramycin concentrations measured remained low $(<2 \mathrm{mg} / \mathrm{L}$ at $24 \mathrm{~h})$ in all patients. Concentration profiles were consistent with a one compartement model with absorption rate-limited first-order release, while showing important variability. A two compartment model was also tested but did not improve the fit. With $\mathrm{CL}$ equated to $\mathrm{CL}_{\mathrm{Cr}}$ mean estimated $\mathrm{k}_{\mathrm{a}}$ was $0.0603 \mathrm{~h}^{-1}$, volume of distribution 16.6 L (CV 89\%) and $\mathrm{F}$ was $63 \%$ and $32 \%$ (CV 74\%) in the 8 patients having received $10 \mathrm{~g}$ Osteoset $^{\circ} \mathrm{T}$ and in the 4 patients having received $20 \mathrm{~g}$, respectively. Sex, age, body weight or implantation site did not seem to affect tobramycin absorption or disposition when introduced as covariates. Population pharmacokinetic parameter estimates and variability are summarized in Table 1 . The concentration values observed are represented on Figure 1, along with mean population prediction and $90 \%$ prediction intervals for $\mathrm{CL}_{\mathrm{Cr}} 120 \mathrm{~mL} / \mathrm{min}$.

The simulations of concentration profiles (using an average $\mathrm{F}$ estimate of $47 \%$ ) showed that more than $5 \%$ patients would maintain concentrations over $2 \mathrm{mg} / \mathrm{L}$ during at least five days post implantation with tobramycin 262, 786 and $1310 \mathrm{mg}$ when $\mathrm{CL}_{\mathrm{cr}}$ was respectively set at 10,20 and $30 \mathrm{~mL} / \mathrm{min}$. 
Table 1 Tobramycin population pharmacokinetic parameter estimates

\begin{tabular}{|c|c|c|}
\hline \multicolumn{3}{|c|}{ Population mean } \\
\hline Parameter & Estimate & s.e $\%^{b}$ \\
\hline$\overline{\mathrm{CL}\left(\mathrm{L} \mathrm{h}^{-1}\right)}$ & $7.14^{d}$ & - \\
\hline$V(L)$ & 16.6 & 35 \\
\hline$K_{a}\left(h^{-1)}\right.$ & 0.0603 & 19 \\
\hline $\mathrm{F}$ (if cast $10 \mathrm{~g}$ ) & 0.63 & 19 \\
\hline $\mathrm{F}$ (if cast $20 \mathrm{~g}$ ) & 0.32 & 19 \\
\hline \multicolumn{3}{|c|}{ Interpatient variability } \\
\hline Parameter & $\mathrm{CV} \%^{a}$ & s.e\% ${ }^{c}$ \\
\hline$\overline{\mathrm{CL}}$ & - & - \\
\hline V & 89 & 74 \\
\hline$k_{a}$ & - & - \\
\hline $\mathrm{F}$ & 74 & 72 \\
\hline \multicolumn{3}{|c|}{ Residual variability } \\
\hline Error type & & s.e\% \\
\hline$\overline{\sigma_{\text {prop }}(C V \%)^{e}}$ & 29 & $50^{c}$ \\
\hline$\sigma_{\text {add }}(S D \text { in } \mathrm{mg} / \mathrm{L})^{f}$ & 0.062 & $22^{b}$ \\
\hline
\end{tabular}

$\mathrm{CL}$, mean apparent clearance; $\mathrm{V}$, mean apparent volume of distribution; $\mathrm{k}_{a}$, mean absorption rate constant; $F$, mean bioavailability.

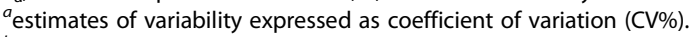

${ }^{b}$ s.e $=$ standard error of the estimates (S.E), defined as S.E/estimate and expressed as percentage.

$c_{\text {s.e }}=$ standard error of the coefficient of variations, taken as

$\sqrt{s . e_{\text {estimate }} / \text { estimate, }}$ expressed as a percentage.

${ }^{d}$ equated to creatinine clearance $\left(\mathrm{CL}_{\mathrm{cr}}\right)$.

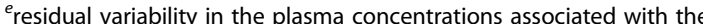
proportional error term, expressed as a coefficient of variation (CV\%). residual variability in the plasma concentrations associated with the additive error term, expressed as a standard deviation (SD).

\section{Discussion and conclusions}

This is the first tobramycin pharmacokinetic study after Osteoset $^{\circ} \mathrm{T}$ implantation in a clinical setting. Tobramycin systemic concentration values were measured well below the traditional toxicity threshold of $2 \mathrm{mg} / \mathrm{L}$ from $24 \mathrm{~h}$ and later on, which was not unexpected considering the low tobramycin dose implanted and the good renal function of the study subjects. Whether continuous systemic exposure to low concentrations below minimal inhibitory concentration may favor antimicrobial resistance remains an issue [6].

Prominent inter-individual variability was observed, probably due to heterogeneity of patients, indications, surgical techniques and implantation sites. There have probably been too few patients included for reliably testing the influence of all covariates. Loss through wound oozing has been neglected (not measured) but could represent another source of variability. Imprecision on low tobramycin levels measurement, very close to the LOQ, could also have increased residual variability.

The significant difference in bioavailability between $10 \mathrm{~g}$ and 20 g Osteoset $^{\circ} \mathrm{T}$ (63\% versus $32 \%$ respectively) could indicate that higher amounts of bone graft substitute either limit tobramycin release to some extent, or slow it down sufficiently for the resulting concentrations to fall below the LOQ. Unbalanced loss through wound oozing could also partly account for this difference. Implantation site and tissue perfusion could also play a role, although this could not be demonstrated in our analysis, due to limited data.

Our model gained in credibility when tobramycin CL was equated to $\mathrm{CL}_{\mathrm{cr}}$; indeed there is no argument to think of a different elimination route for the drug absorbed from Osteoset ${ }^{\circ} \mathrm{T}$ compared to intravenous delivery [7]. The Cockcroft equation that we used has yet its limitations, in particular in obese or bedridden patients. Our model found a distribution volume of $0.22 \mathrm{~L} / \mathrm{kg}$ body weight, consistent with previously published values [7]. The large inter-patient variability found in both $\mathrm{V}$ and $\mathrm{F}$ is likely to incorporate some amount of variability in CL and $k_{a}$.

High sustained potentially toxic concentrations were predicted by simulations with standard doses Osteoset ${ }^{\circ}$
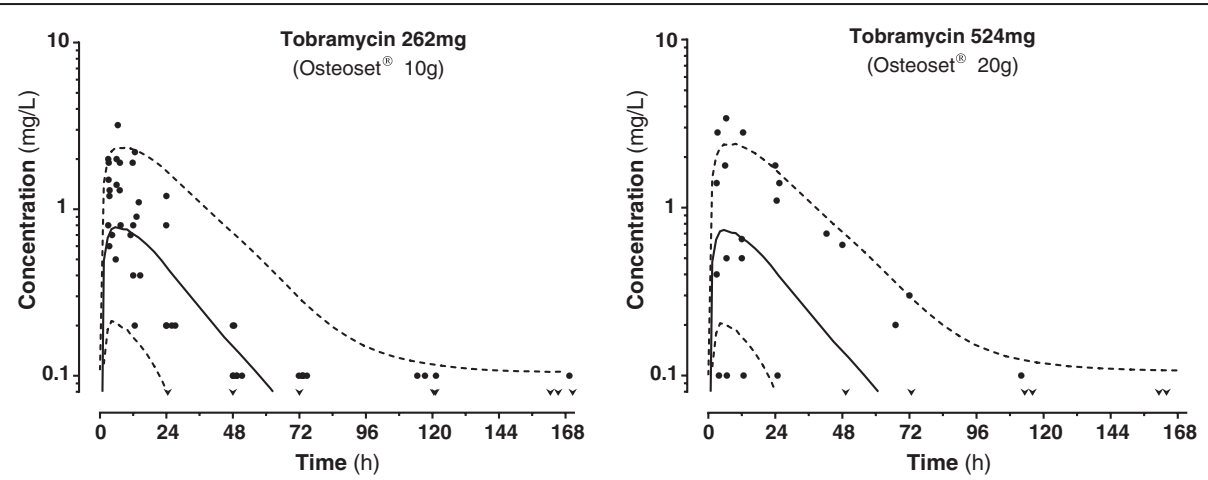

Figure 1 Concentrations versus time plot of tobramycin with mean population prediction (solid line) and $90 \%$ prediction interval (dashed lines) after Osteoset ${ }^{\circledR} \mathbf{1 0 ~ g}$ (left) or $\mathbf{2 0} \mathrm{g}$ (right) in patients with normal renal function. Dose difference is almost fully compensated by different bioavailability, giving similar predictions. Superimposed points show the concentrations observed in 8 patients after $10 \mathrm{~g}$ and 4 patients

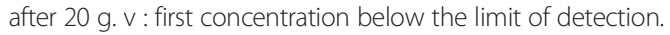


$\mathrm{T}$ at renal failure stages 4 and 5 . Considering the limited study power, this simulation should be taken as a rough estimate of the potential for toxicity of this product. Our model is probably too imprecise to deduce precise dose adjustment guidelines. However, it indicates that caution is warranted when Osteoset ${ }^{\ominus} \mathrm{T}$ implant is considered for patients with severe renal failure, as its benefit/risk ratio could turn out unfavourable [8].

\section{Competing interests}

The authors declare that they have no competing interests.

\section{Authors' contributions}

FL performed the pharmacokinetic analysis and was involved in drafting the manuscript. PW designed the study and was involved in the acquisition of data. CC revised the pharmacokinetic analysis. EG was involved in the study design and coordination. TB gave final approval of the version to be published. All authors read and approved the final manuscript.

\section{Acknowledgements}

We thank Ali Maghraoui for technical assistance.

\section{Author details}

'Division of Clinical Pharmacology, Biomedicine, Department of Laboratories, Centre Hospitalier Universitaire Vaudois, Lausanne 1011, Switzerland.

${ }^{2}$ Department of Orthopedic Surgery, Cantonal Hospital, Fribourg, Switzerland. ${ }^{3}$ Department of Pharmaceutical Sciences, Clinical Pharmacy Unit, University of Geneva, Rue du Général- Dufour 24, Genève 4 1211, Switzerland.

Received: 22 August 2013 Accepted: 20 February 2014

Published: 4 March 2014

\section{References}

1. Nelson CL, McLaren SG, Skinner RA, Smeltzer MS, Thomas JR, Olsen KM: The treatment of experimental osteomyelitis by surgical debridement and the implantation of calcium sulfate tobramycin pellets. J Orthop Res 2002, 20:643-647.

2. Sterling GJ, Crawford S, Potter JH, Koerbin G, Crawford R: The Pharmacokinetics of Simplex-tobramycin bone cement. J Bone Joint Surg 2003, 85-B:646-649.

3. Beal SL, Sheiner LB, Boeckmann AJ: NONMEM Users Guides 1989-2006. Ellicott City, Maryland, USA: Eds Icon Development Solutions.

4. Cockcroft DW, Gault MH: Prediction of creatinine clearance from serum creatinine. Nephron 1976, 16:31-41.

5. Wahl P, Livio F, Jacobi M, Gautier E, Buclin T: Systemic exposure to tobramycin after local antibiotic treatment with calcium sulfate as carrier material. Arch Orthop Trauma Surg 2011, 131:657-662.

6. Freeman CD, Nicolau DP, Belliveau PP, Nightingale CH: Once-daily dosing of aminoglycosides: review and recommendations for clinical practice. J Antimicrob Chemother 1997, 39:677-686.

7. Burton ME, Shaw LM, Schentag JJ, Evans WE: Aminoglycosides. In Applied Pharmacokinetics and Pharmacodynamics - Principles of Therapeutic Drug Monitoring. 4th edition. Edited by Troy D, Hauber M, Remsberg C. Philadelphia: Lippincott Williams and Wilkins; 2006:285-327.

8. Wu IM, Marin EP, Kashgarian M, Brewster UC: A case of an acute kidney injury secondary to an implanted aminoglycoside. Kidney Int 2009, 75:1109-1112.

doi:10.1186/2050-6511-15-12

Cite this article as: Livio et al:: Tobramycin exposure from active calcium sulfate bone graft substitute. BMC Pharmacology and Toxicology 2014 15:12.

\section{Submit your next manuscript to BioMed Central and take full advantage of:}

- Convenient online submission

- Thorough peer review

- No space constraints or color figure charges

- Immediate publication on acceptance

- Inclusion in PubMed, CAS, Scopus and Google Scholar

- Research which is freely available for redistribution 